\title{
Tolosa Hunt syndrome followed by facial nerve palsy
}

\section{Rawan Alnajashi ${ }^{1}$, Hind Alnajashi²}

1. Department of Medicine, College of Medicine, King Abdulaziz University, Jeddah, Kingdom of Saudi Arabia

2. Department of Medicine, Neurology Division, King Abdulaziz University, Jeddah, Kingdom of Saudi Arabia

\section{CASE STUDY}

Please cite this paper as: Alnajashi R, Alnajashi H. Tolosa Hunt syndrome followed by facial nerve palsy. AMJ 2020;13(1):1-3.

https://doi.org/10.35841/1836-1935.13.1.1-3

\section{Corresponding Author:}

Rawan Alnajashi

Department of Medicine, College of medicine, King Abdulaziz University, Jeddah, Saudi Arabia

Email: najrawan@gmail.com

\section{ABSTRACT}

Tolosa Hunt syndrome (THS) is a rare neurological syndrome, manifesting as a unilateral headache with painful ophthalmoplegia. THS main symptoms are thought to be derived from the granulomatous inflammation of the cavernous sinus, superior orbital fissure or orbits. Although the facial nerve does not originate from the cavernous, few case reports have observed the involvement of facial nerve, proposing the idea of a diffuse systemic inflammation. We here describe a case of a typical THS in the form of initial presentation, imaging and response to treatment but has atypical features, in the form of extracavernous cranial nerve involvement.

\section{Key Words}

Tolosa Hunt syndrome, ophthalmoplegia, facial nerve palsy

\section{Implications for Practice:}

\section{What is known about this subject?}

Tolosa Hunt syndrome is a rare disorder usually limited to the cranial nerves in the cavernous sinus and orbital fissure.

\section{What new information is offered in this case study?}

The Facial nerve involvement reported in our case is atypical and indicates a more systemic inflammation. Reporting such cases is necessary for understating the pathology.

3. What are the implications for research, policy, or practice?

Reporting atypical cases of THS with extracavernous involvement help in expanding our understanding and change the diagnostic criteria.

\section{Background}

Tolosa Hunt syndrome (THS) is a rare disorder with an incidence of one per million. ${ }^{1}$ The underlying cause is assumed to be idiopathic inflammation of the cavernous sinus or orbital apex. ${ }^{2}$ This inflammation causes painful cranial nerve palsy involving all or some of the following nerves: the third, fourth or sixth cranial nerves, V1 and/or V2 divisions of the fifth cranial nerve. ${ }^{3}$ Here we describe a case of THS that has atypical clinical presentation, in the form of extracavernous cranial nerve involvement.

\section{Case details}

A 26-year-old female patient presented with a one-month history of severe right-sided throbbing headache and double vision that was getting gradually worse. At emergency room assessment, Blood Pressure was 149/90. Cranial nerve examination showed normal vision acuity $20 / 20$, bilateral equal and reactive pupil to light, normal fundus exam and visual field. There was complete ptosis of the right eye, and ophthalmoplegia of the third, fourth and sixth cranial nerves. With decreased facial sensation on V1, V2 trigeminal nerve branches on the right side. The rest of the neurological exam was normal. The patient was suspected to have cavernous sinus pathology. Urgent Magnetic resonance imaging (MRI) and magnetic resonance venography of the brain and orbits were obtained. MRI showed asymmetric fullness and intense post-contrast enhancement of the right cavernous sinus, extending through the superior orbital fissure to the orbital apex with 
no evidence of venous thrombosis (Figure 1). These findings were suggestive of Tolosa Hunt syndrome. The cerebrospinal fluid study showed normal protein $0.39 \mathrm{~g} / \mathrm{L}$, normal glucose $4 \mathrm{mmol} / \mathrm{L}$ and $W B C 6$ per cubic millimetre, negative gram stain, and cultures. ANA, thyroid function test, vitamin B12 and ESR were normal. She was started on high dose pulse steroid therapy $1000 \mathrm{mg}$ for three days followed by oral prednisolone $60 \mathrm{mg}$ per day with slow tapering. Her headache improved significantly within three days of starting steroid treatment. On follow up assessment three weeks later she had complete resolution of the headache and ophthalmoplegia. She was kept on slowly tarping doses of oral prednisolone. Two months later after reaching a dose of $15 \mathrm{mg}$ she experienced a new headache with a left Lower motor neurone facial palsy. She was treated with acyclovir and high dose oral prednisolone tapered over two weeks. Headache improved but she still has a mild residual left facial weakness. Repeated MRI brain (Figure 2) was normal with complete resolution of previous cavernous sinuous abnormality.

\section{Discussion}

Tolosa Hunt syndrome (THS) is an idiopathic inflammation of the cavernous sinus or orbital apex, which results in painful ophthalmoplegia and facial pain secondary to trigeminal nerve involvement. ${ }^{2,4}$ The most important step in the diagnosis is to exclude the more serious pathologies such as cavernous venous thrombosis, infections or compressive tumour. The Headache classification committee of the International Headache Society diagnostic criteria -Third edition ICHD-3 suggest the following diagnostic criteria for Tolosa-Hunt syndrome: ${ }^{5}$

A. Unilateral orbital or periorbital headache fulfilling the following criteria:

1. headache on the same side of the granulomatous inflammation.

2. headache has preceded the onset of cranial nerve palsy by 2 weeks or less than 2 weeks or developed with it.

B. Both of the following:

1. granulomatous inflammation of the cavernous sinus, superior orbital fissure or orbits, demonstrated by MRI or biopsy.

2. paresis of one or more of the ipsilateral third, fourth and/or fifth cranial nerves.

C. Not better accounted for by another ICHD-3 diagnosis. Rapid resolution of the pain within two to three days from starting the steroid therapy is a characteristic feature that can help to confirm the diagnosis ${ }^{3,6}$ although other causes can respond to steroid initially, The improvement of cranial nerve palsy and radiological changes can take a longer time. ${ }^{1,3}$ Few case reports described the rare association of extracavernous cranial nerve involvement in THS suggesting a more diffuse systemic inflammation. ${ }^{7-11} \mathrm{~A}$ case series of 14 cases of THS mentioned the involvement of facial nerve in four cases. Three cases developed facial nerve palsy after recovery from THS and one case had facial nerve palsy before the onset of THS; of these cases, two had involvement of facial nerve in the contralateral side of THS. $^{11}$ Recurrent facial nerve palsy proceeding the onset of THS has been reported in one case. ${ }^{8}$ We describe a typical case of THS based on the clinical presentation, MRI finding and improvement with steroid treatment. However, the facial nerve palsy shortly following this clinical event is atypical and can be explained by two hypotheses: First, it can be an incidental idiopathic facial nerve palsy unrelated to the THS. Second, it could suggest that THS is a part of an idiopathic inflammation of the cranial nerve inflammation not limited to the cavernous sinus. Even though our hypotheses could be true or not, reporting such atypical cases of THS could help in expanding our knowledge and the understanding of this idiopathic cranial nerve inflammation or finding an underlying cause. We stress on the fact that THS remains a diagnosis of exclusion. Many inflammatory and neoplastic conditions can have a similar presentation and response to steroid. Therefore, an extensive workup should be done to exclude other cause before making the diagnosis.

\section{Conclusion}

THS is a rare disorder that is not fully understood. Reporting atypical cases of THS could further expand our understanding and adds to the literature.

\section{References}

1. Zurawski J, Akhondi H. Tolosa-Hunt syndrome - A rare cause of headache and ophthalmoplegia. Lancet. 2013;382(9895):912.

2. Hung $\mathrm{CH}$, Chang $\mathrm{KH}$, Chen $\mathrm{YL}$, et al. Clinical and radiological findings suggesting disorders other than Tolosa-Hunt syndrome among ophthalmoplegic patients: A retrospective analysis. Headache. 2015;55(2):252-64

3. Arshad A, Nabi S, Panhwar MS, et al. Tolosa-Hunt syndrome: An arcane pathology of cavernous venous sinus. BMJ Case Rep. 2015;2015:bcr2015210646.

4. Khan ZA, Ahmed Siddiqui A, Syed J, et al. Clinical insights on Tolosa Hunt syndrome: A multidisciplinary approach on neurological-related symptomatology in maxillofacial region. AMJ. 2018;11(2):143-7.

5. Vincent M, Wang S. Headache Classification Committee of the International Headache Society (IHS) The 
International Classification of Headache Disorders, 3rd edition. Cephalalgia. 2018;38(1):1-211.

6. Hung $\mathrm{CH}$, Chang $\mathrm{KH}, \mathrm{Wu} \mathrm{YM}$, et al. A comparison of benign and inflammatory manifestations of Tolosa-Hunt syndrome. Cephalalgia. 2013;33(10):842-52.

7. Tessitore E, Tessitore A. Tolosa-Hunt Syndrome Preceded by Facial Palsy. Headache. 2000;40(5):393-6.

8. Shahrizaila N, Ramli N, Tan CT. Tolosa-Hunt syndrome following recurrent facial palsies. Neurology Asia. 2010;15(1):97-9.

9. Kang H, Park KJ, Son S, et al. MRI in Tolosa-Hunt Syndrome Associated with Facial Nerve Palsy. Headache. 2006;46(2):336-9.

10. Pirastehfar M, Luo J. Tolosa-Hunt syndrome with facial nerve involvement (P1.298). Neurology. 2015;84(14 Supplement).

11. Miwa H, Koshimura I, Mizuno Y. Recurrent cranial neuropathy as a clinical presentation of idiopathic inflammation of the dura mater: A possible relationship to Tolosa-Hunt syndrome and cranial pachymeningitis. J Neurol Sci. 1998;154(1):101-5.

\section{PEER REVIEW}

Not commissioned. Externally peer reviewed.

\section{CONFLICTS OF INTEREST}

The authors declare that they have no competing interests.
Figure 1: (A) axial and (B) coronal section of T1 MRI with contrast showing asymmetric fullness and intense enhancement of the right cavernous sinus, extending through the superior orbital fissure to the orbital apex

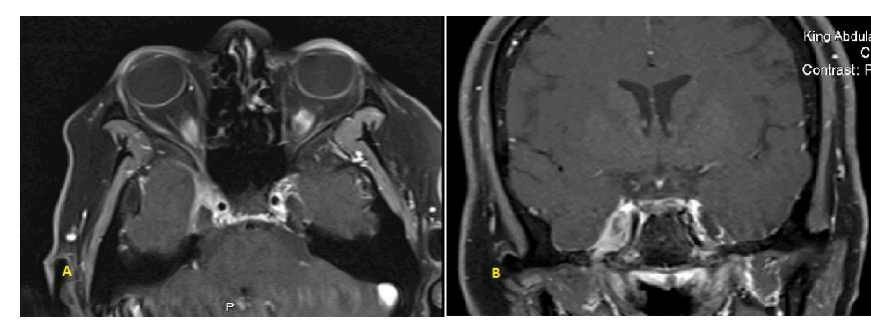

Figure 2: (A) axial T1 with contrast and axial T2 MRI brain showed resolution of the asymmetric thickening and enhancement involving the right cavernous sinus and superior orbital fissure
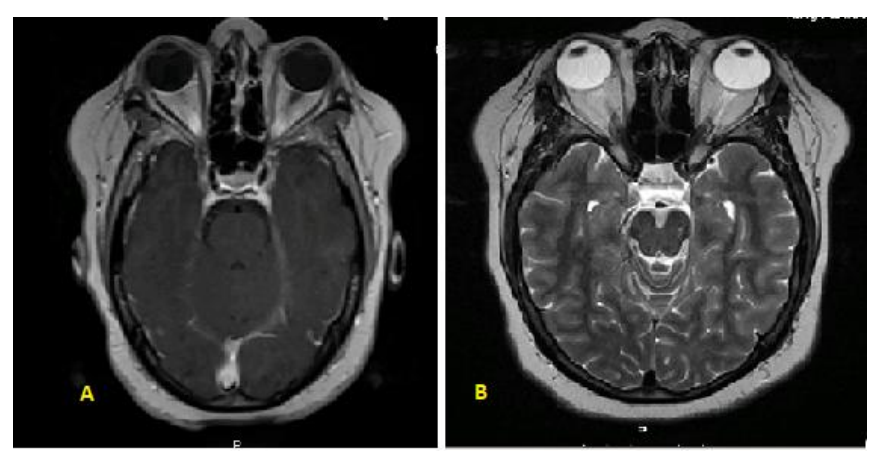

\section{FUNDING}

None

\section{PATIENT CONSENT}

The authors, Alnajashi $R$, Alnajashi $H$, declare that:

1. They have obtained written, informed consent for the publication of the details relating to the patient(s) in this report.

2. All possible steps have been taken to safeguard the identity of the patient(s).

3. This submission is compliant with the requirements of local research ethics committees. 